\title{
Preparación prequirúrgica de la piel con clorhexidina al 2\% como factor de prevención de la infección en el sitio quirúrgico ${ }^{1}$
}

Evelin Solano Castro ${ }^{2}$

Institución: Hospital Nacional de Niños Dr. Carlos Sáenz Herrera

\section{CÓMO CITAR}

Solano, E. (Abril, 2014). Preparación prequirúrgica de la piel con clorhexidina al $2 \%$ como factor de prevención de la infección en el sitio quirúrgico. Rev. Actual de Costa Rica, 26, 1-15. Recuperado de <http://www.revenf.ucr.ac.cr/prepaquiru.pdf> ISSN 1409-4568

\section{RESUMEN}

En este trabajo se presentan los resultados de una investigación secundaria acerca de la preparación prequirúrgica de la piel con el antiséptico clorhexidina al 2\%. Las Infecciones del sitio quirúrgico son una de las complicaciones más comunes en los procedimientos quirúrgicos y se asocian con una morbilidad y mortalidad significativas en el usuario (a), aparte de que son la tercera infección más frecuente asociada a la atención de salud. Se aplicaron los pasos de la práctica clínica basada en la evidencia: en primera instancia se planteó una pregunta en formato PICO, y luego se efectuó una búsqueda de información en las bases de datos recomendadas en el Curso de Práctica Clínica de Enfermería Basada en la Evidencia, impartido por el Programa de Colaboración para la Investigación en Enfermería Basada en la Evidencia de Costa Rica (CIEBE-CR). Se consultó las bases PubMed, Crochane LIBRARY, National Center for Biotechnology Information (NCBI), Google Académico, CINAHL (Cummulative Index of Nursing and Allied Health Literature). SCIELO (Scientific Electronic Library on line www.Scielo.org. Se recuperó 22 documentos de los cuales solo tres cumplían con rigurosidad metodológica. Para el análisis crítico se utilizó el software Fichas de Lectura Crítica 2.0 (FLC). Se concluye que la clorhexidina al 2\%, es la mejor opción como antiséptico para al preparar la piel de manera prequirúrgica ; sin embargo, es necesario desarrollar más estudios con el objetivo de determinar cuál es la forma correcta en cuanto a concentración, frecuencia, técnica y efectos adversos en la población pediátrica.

Palabras claves: cirugía-cardíaca, gluconato-de-clorhexidina-2\%, infecciones-del-sitio-quirúrgico, preparaciónprequirúrgica-de-la-piel.

\footnotetext{
${ }^{1}$ Fecha de recepción: 25 de noviembre del 2013

Fecha de aceptación: 13 de marzo del 2014

${ }^{2}$ Enfermera. Hospital Nacional de Niños Dr. Carlos Sáenz Herrera. Correo electrónico: evelinsolanocastro@yahoo.es
}

${ }^{1}$ Date of receipt: november 25, 2013 


\title{
Preoperative skin preparation with $2 \%$ chlorhexidine as a factor in the prevention of surgical site infection ${ }^{1}$
}

Evelin Solano Castro ${ }^{2}$

Institution: Dr. Carlos Sáenz Herrera, National Children's Hospital

CITED

Solano, E. (April, 2014). Preoperative skin preparation with $2 \%$ chlorhexidine as a factor in the prevention of surgical site infection. Rev. Actual de Costa Rica, 26, 1-15. Recuperado de <http://www.revenf.ucr.ac.cr/prepaquiru.pdf> ISSN 1409-4568

\begin{abstract}
The results of secondary research that refers to preoperative skin preparation with antiseptic chlorhexidine $2 \%$ are presented. Surgical Site Infections are one of the most common complications in surgical procedures are associated with significant morbidity and mortality in the user and are the third -associated infection more frequent in the health care. Steps of clinical practice based on evidence were applied, considering in the first instance a question in PICO format, then a search for information in databases recommended in the Course of Clinical Nursing Practice Evidence-Based, taught by the program for Collaborative Research in Evidence-Based Nursing of Costa Rica ( CIEBE -CR ). The PubMed database and Cochrane LIBRARY was consulted, National Center for Biotechnology Information ( NCBI), Google Scholar, CINAHL (cummulative Index of Nursing and Allied Health Literature). SCIELO (Scientific Electronic Library on line www.Scielo.org . 22 documents were recovered, but only three were selected because had methodological rigor. For the critical analysis Critical Reading Sheets 2.0 ( FLC) software was used. Was concluded that $2 \%$ chlorhexidine, is the best choice for preoperative skin preparation antiseptic, however, it is necessary to conduct further studies in order to determine which is the correct way in strength, frequency, technical and adverse effects in the pediatric population.
\end{abstract}

Keywords: cardiac-surgery, gluconate-chlorhexidine $2 \%$, preoperative, skin-preparation, surgical-siteinfections.

\footnotetext{
${ }^{1}$ Date of receipt: november 25, 2013

Date of acceptance: march 13, 2014

${ }^{2}$ Nurse. Dr. Carlos Sáenz Herrera, National Children's Hospital. E mail: evelinsolanocastro@yahoo.es
} 


\section{INTRODUCCIÓN}

Las infecciones nosocomiales están relacionadas con las prácticas clínicas asistenciales en usuarios (a) hospitalizados o ambulatorios que tienen contacto con el sistema de salud; por lo general, afectan al 5\% de los pacientes y significan un aumento en la morbimortalidad quirúrgica, un mayor costo económico y una menor calidad en la atención de la salud (Cantón, Viudes y Peman, 2001), de hecho, según Pujol y Limón (2013), "los principales tipos de infección relacionada con el sistema sanitario están relacionados con procedimientos invasivos y son la infección respiratoria, la quirúrgica, la urinaria y la bacteriemia de catéter vascular” (p.1)

Las infecciones varían en el orden de clasificación por aparición y en los servicios quirúrgicos y representan la mayor de las complicaciones de un procedimiento quirúrgico; según estudios, constituyen "la tercera infección nosocomial más frecuente (14-16\%) y la primera entre los pacientes quirúrgicos (38\%)" (Aizcorbe, Izco, De la Torre, Usoz y Soto, 2000, p.130); según Bagnail (2009), citado por Silva (2013), "las infecciones del sitio quirúrgico son las infecciones más comunes adquiridas en los hospitales”. (p.78). No obstante, en la actualidad representan la segunda causa más común (Pujol y Limón, 2013, p.p. 108-113), aun cuando son un evento adverso prevenible de la cirugía que incrementa los gastos de la hospitalización.

En específico, las infecciones del sitio quirúrgico, según Burke, H., Derek, B., BAO, J., Ni-Dhonochu, T., Shields, W., Desmond, C. y Mealy, K. (2010), "son aquellas que ocurren en una herida operatoria dentro de los 30 días del procedimiento o dentro del año, si se implantó una prótesis”(p. 1).

En cuanto a su distribución en América Latina, en Cuba de acuerdo con Alemán y Guanche (2010) , el $18,5 \%$ de las infecciones nosocomiales fueron del sitio quirúrgico.

En el caso de Costa Rica, tanto en hospitales generales como en el pediátrico, se presenta una alta incidencia de infecciones del sitio quirúrgico, dado que para el 2005, el Comité de Prevención y Control de Infecciones Hospitalarias del Hospital Dr. Rafael Ángel Calderón Guardia en el 2006 documentó un porcentaje global del 11.5\% de infecciones hospitalarias, de las cuales el 34\% correspondieron a infección de herida operatoria. En el Hospital Nacional de Niños, la incidencia de infecciones nosocomiales en el año 2012 alcanzó un 4.6\%; de ese porcentaje el 7\% representa infecciones en el sitio quirúrgico (CCPIN, 2013). Luego, en el servicio de la unidad cardíaca, en el primer semestre del 2013, el 7.8\% de las infecciones nosocomiales correspondieron a infecciones en el sitio quirúrgico; si se comparan cifras se nota diferencia en los porcentajes; sin embargo, la infección del sitio quirúrgico continúa siendo una de las tres primeras causas de las infecciones nosocomiales.

De los datos anteriores se colige que la infección del sitio quirúrgico es una complicación esperable en cualquier acto quirúrgico por la vulnerabilidad que supone la descontinuación de la integridad de la piel por la incisión quirúrgica, razón que motiva a unir esfuerzos para disminuir dicho efecto adverso que afecta la seguridad de la atención en salud.

En el mundo, existen diversos organismos encargados de la vigilancia en lo que respecta a infecciones nosocomiales, entre ellos se destaca el Centers for Disease Control and Prevention (CDC) de Estado Unidos, la primera agencia de promoción de la salud, prevención y preparación del país y una agencia líder mundial en el 
área de la salud pública (CDC, 2013, p. 3); por consiguiente, el CDC es la organización mundial que más ha desarrollado estudios clínicos para elaborar guías clínicas con el fin de prevenir las infecciones nosocomiales. Al respecto, el CDC afirma que diversos factores determinan el riesgo de una infección del sitio quirúrgico, la mayoría son prevenibles, motivo por el que desarrollaron la guía para prevenir la infección del sitio quirúrgico (Mangram, A., Horan, T., Pearson, M., Silver, L. y Jarvis, W.,1999) en la que mencionan varios factores de riesgo prevenibles, divididos en grupos; por ejemplo, por las características del grupo 1, es necesaria la preparación de la piel del paciente con una ducha antiséptica preoperatoria.

Según la experiencia de la investigadora, la preparación prequirúrgica de la piel con antiséptico en el Hospital Nacional de Niños (HNN) no está estandarizada y, en la mayoría de los casos, no existe suficiente evidencia científica, puesto que desde el inicio ha tenido un nivel de evidencia tipo III "fundamentado en opiniones basadas en experiencias clínicas" y hasta la fecha, no existe algún escrito que aporte un nivel de confianza mejor, por lo que su grado de recomendación es I; es decir, "la evidencia es insuficiente, de mala calidad o contradictoria, y el balance entre beneficios y perjuicios no puede ser determinado" (Harris, R., Helfand, M., Woolf, S., Lohr, K., Mulrow, C., Teutsch, S. y Atkins, D., 2001).

Debido al panorama mencionado, se planteó esta investigación desde el curso de Práctica Clínica de Enfermería Basada en la Evidencia impartido por el programa CIEBE-CR, el cual brinda a "la enfermera y el enfermero clínico y gestor, los instrumentos para cuestionarse los cuidados que realiza, y lo más importante, los capacita para responder a sus preguntas e introducir cambios en su práctica por medio de la investigación científica basada en la evidencia (Rojas, Cubero y Rodríguez, 2013, p.1).

El objetivo de este trabajo consiste en determinar el grado de confiabilidad de la evidencia científica en la preparación prequirúrgica de la piel con antiséptico con clorhexidina $2 \%$, para los niños(as) sometidos a cirugías cardíacas, con el fin de poder establecer criterios de eficacia, seguridad y tolerancia que faciliten la toma de decisiones acerca de la mejor de las acciones para disminuir las infecciones del sitio quirúrgico.

\section{MATERIALES Y MÉTODOS}

Para alcanzar el objetivo de este estudio se utilizó lo aprendido en la práctica de enfermería basada en la evidencia, impartida en el Curso de Práctica Clínica de Enfermería Basada en la Evidencia, impartido por el programa CIEBE-CR. Dicha metodología incluye 5 pasos:

1. Formulación de una pregunta de primera línea en el formato PICO.

2. Búsqueda de información científica disponible.

3. Análisis crítico de los datos.

4. Implementación de los resultados encontrados en el quehacer diario del profesional.

5. Evaluación de la implementación. 


\section{Paso 1. Formulación de la pregunta PICO}

Esta pregunta debe tener una estructura en la que se identifiquen claramente la persona o población o situación problema, la intervención habitual frente al problema, la intervención nueva por considerar y, por último, el efecto o resultado que se espera.

Dicha pregunta se formuló como respuesta a este primer paso con el formato PICO de la siguiente manera:

Tipo de paciente o la patología de la que surge la pregunta (P)

La intervención que se desea analizar (I)

La comparación con otra intervención (si procede) (C)

Los resultados clínicos esperados $(\mathrm{O})$

Se redactó una pregunta clínica de primera línea de tipo de intervención de la siguiente forma:

En los pacientes pediátricos sometidos a una cirugía cardíaca, ¿la preparación prequirúrgica de la piel con baño de clorhexidina al $2 \%$, comparado con la no preparación de la piel, disminuye las infecciones en el sitio quirúrgico?

\section{Paso 2. Búsqueda de información científica disponible}

Posteriormente, se realizó una búsqueda de información en las bases de datos revisadas en el Curso de Práctica Clínica de Enfermería Basada en la Evidencia, impartido por CIEBE-CR.

Las fuentes de datos consultadas fueron las bases Pub Med, Crochane LIBRARY, National Center for Biotechnology Information (NCBI), Google Académico, CINAHL (Cummulative Index of Nursing and Allied Health Literature) y SCIELO (Scientific Electronic Library on line www.Scielo.org de datos electrónicas.

Los criterios de búsqueda se limitaron a ensayos clínicos, estudios de cohortes y revisiones sistemáticas.

Entre los criterios de exclusión se consideró los estudios con sesgos metodológicos, sesgos de publicación, los que presentaran poblaciones de adultos o mixtas, dado que por tratarse de niños, y considerando las características especiales de su piel, las condiciones de vulnerabilidad son diferentes.

Se utilizaron los descriptores preparación AND prequirúrgica AND piel AND niños AND cirugía AND cardiaca AND infección AND sitio AND quirúrgico en español y en inglés.

\section{Paso 3. Análisis crítico de los datos}

El análisis crítico de los datos permite adquirir las habilidades necesarias para excluir con la mayor prontitud los artículos científicos de mala calidad y aceptar aquellos otros con la suficiente calidad científica para ayudar en la toma de decisiones para el cuidado de los pacientes (Red Caspe.org, 2013). Lo anterior influyó para definir qué artículos suministraban la mejor información; de los 22 artículos recuperados y relacionados con el tema, solo tres se consideraron para desarrollar el análisis crítico, los cuales brindaron suficiente evidencia acerca 
del uso de la clorhexidina al $2 \%$, específicamente como preparación prequirúrgica de la piel con antiséptico y su impacto al disminuir la aparición de las infecciones del sitio quirúrgico en comparación respecto del uso del jabón de tocador.

\section{Paso 4. Implementar los resultados encontrados en el quehacer diario del profesional}

En esta etapa se consideró presentar los resultados ante la Jefatura del Departamento de Cirugía y de Enfermería del Hospital Nacional de Niños, tanto a la Dirección como al Departamento de Educación, con el fin de que se tomen decisiones en conjunto en cuanto a a la preparación prequirúrgica, considerando los recursos materiales y humanos a la hora de implementar los hallazgos.

\section{Paso 5. Evaluación de la implementación}

Para ejecutar el estudio, se llevará un registro, de noviembre 2013 a noviembre 2014, de los niños (as) que sean sometidos a cirugía cardíaca y se les aplicará la preparación prequirúrgica de la piel con clorhexidina al 2\%, en el área del tronco, dos veces antes de la cirugía como mínimo, la noche previa a la cirugía, y la mañana antes de la cirugía (aplicadas preferiblemente por el equipo de salud). Los resultados se compararán con el de niños (as) que vengan de otros hospitales y no hayan cumplido con el protocolo de preparación y con las cirugías de emergencia.

Cada niño será valorado en grupos de acuerdo con la edad: el grupo A incluye niños de 0 meses a un año; el $\mathrm{B}$, de un año a 7años y el grupo $\mathrm{C}$, mayores de 7años. Esta agrupación es necesaria al respecto de la concentración de la clorhexidina vrs efectividad según edad y efectos adversos.

Para evaluar la efectividad del baño prequirúrgico con clorhexidina al $2 \%$, se monitorizará los pacientes hasta 40 días después de la cirugía, se tomará como efectivo la no aparición de infecciones del sitio quirúrgico.

\section{RESULTADOS}

En diciembre del 2009, Dizer, Hatipoglu, Kaymakcioglu, Tufan, Yava, Iyigun \& Senses, un grupo de enfermeras publicó en "The effect of nurse-performed preoperative skin preparation on postoperative surgical site infections in abdominal surgery", artículo en el que, por medio de un ensayo clínico, buscaban determinar el efecto de los procedimientos de preparación preoperatoria de la piel realizado por enfermeras para disminuir la infección del sitio quirúrgico en cirugía abdominal.

Los procedimientos desarrollados para la preparación preoperatoria de la piel aplicadas por la enfermera fueron probados en un grupo control $(n=39)$ y un grupo de estudio $(n=43)$.

En los pacientes del grupo control solo se aplicaron los procedimientos rutinarios para la preparación preoperatoria de la piel, mientras que en los miembros del grupo de estudio se empleó el procedimiento de preparación preoperatoria de la piel. Como un requisito del procedimiento, todos los miembros del grupo de estudio tenían un baño de clorhexidina al menos dos veces después de su hospitalización y al menos una vez una noche antes de la operación, bajo condiciones controladas. 
De los resultados se pudo observar que en el grupo en el que no se aplicó baño de clorhexidina, el riesgo de infección fue 4.76 veces $(95 \%$ CI $=1.20-18.83)$ más que en el grupo en el que sí se aplicó, incluso, después de las correcciones, considerando la edad y género. La diferencia entre el grupo control y grupo de estudio respecto de las infecciones del sitio quirúrgico también fue estadísticamente significativa $(\mathrm{p}<0.05)$.

Una de las características destacables de este estudio es que fue la enfermera quien preparó la piel de los usuarios de la forma adecuada, y en el tiempo adecuado, en el total del grupo de investigación, situación muy significativa en cuanto al riesgo de aparición de las infecciones en el sitio quirúrgico, si se compara con el resto de investigaciones.

En setiembre del 2011, en la Journal Shoulder and Elbow Surgery, Murray, Saltzman, Gryzlo, Terry, Woodward y Nuber publicaron " Efficacy of preoperative home use of $2 \%$ chlorhexidine gluconate cloth before shoulder surgery". En este estudio se evaluó la hipótesis de que el uso casero de paños de gluconato de clorhexidina al $2 \%$, antes de la cirugía de hombro, es más eficaz que una ducha estándar de agua y jabón para disminuir los niveles de bacterias patógenas de la flora cutánea preoperatoria.

Entre enero de 2010 y mayo de 2010, se seleccionaron 100 pacientes consecutivos que serían sometidos a una cirugía de hombro, para el estudio prospectivo aleatorizado. Los criterios de inclusión fueron pacientes programados para cualquier tipo de cirugía de hombro. Todos los procedimientos se llevaron a cabo en una sola institución, se asignó en sobres el grupo control y el grupo de tratamiento; por ende, los cirujanos no sabían el grupo al que pertenecía cada paciente. A los pacientes asignados al grupo de tratamiento se enviaron 2 juegos de paños impregnados con gluconato de clorhexidina $2 \%$. Estos pacientes fueron instruidos para ducharse con agua y jabón la noche antes de su operación y para limpiar la totalidad de su extremidad operativa, incluyendo la axila, hombros, pecho ipsilateral y la espalda, con un paño impregnado con gluconato de clorhexidina $2 \%$.

La mañana de la cirugía, los pacientes del grupo de tratamiento debían aplicar un segundo paño impregnado con gluconato de clorhexidina $2 \%$, de la misma manera que el primero, dentro de las 2 horas previas a su ingreso al hospital. Los pacientes asignados al grupo de control recibieron instrucciones para ducharse con agua y jabón.

La mañana de la cirugía, al llegar a la sala de espera preoperatoria, completaron un cuestionario que incluía la edad, la capacidad para completar el protocolo de limpieza preoperatoria, la ocurrencia de efectos adversos o reacciones a las telas de gluconato de clorhexidina; luego se tomaron muestras y análisis de la flora bacteriana.

Los pacientes además fueron monitorizados durante 2 meses luego de la operación para detectar signos clínicos de infección.

Entre de los resultados se evaluó que en el grupo de tratamiento versus el grupo control, la tasa global de cultivos positivos fue del $66 \%$ vs $94 \%$ ( $p=0,0008$ ), y la tasa de cultivos positivos para Staphylococcus coagulasa negativo fue $30 \%$ vs $70 \%(p=0,0001)$.

No se produjeron infecciones en ninguno de los pacientes en un mínimo de 2 meses después de la cirugía. 
De dicho estudio se concluyó que el uso de la tela de clorhexidina al $2 \%$ fue eficaz para disminuir las tasas globales de cultivo bacteriano, antes de la cirugía de hombro, y su eficacia era aun más notable para reducir la cantidad de Staphylococcus coagulasa-negativa; por ende, puede ser un complemento útil como estrategias de prevención de infecciones del sitio quirúrgico. Al respecto, es necesario recalcar que este fue un estudio con Nivel de evidencia I, ensayo controlado aleatorio, estudio de tratamiento, en el que se considera la desinfección de casi las mismas áreas que se deben abarcar para la preparación prequirúrgica de cirugía cardiaca.

En febrero del 2013, Chlebicki, Safdar, O'Horo y Maki publicaron "Preoperative chlorhexidine shower or bath for prevention of surgical site infection: a meta-analysis", estudio en el que efectuaron una revisión sistemática y su respectivo metaanálisis de ensayos controlados prospectivos hasta el 2011 para determinar la efectividad del baño preoperatorio con clorhexidina versus placebo o ningún baño para prevenir la infección del sitio quirúrgico, debido a que para los investigadores el baño con clorhexidina es frecuentemente recomendado como una medida preoperatoria para prevenir la infección del sitio quirúrgico, sin embargo, la eficacia de este enfoque fue incierto. Los investigadores realizaron una búsqueda de bases de datos electrónicas en MEDLINE, incluyendo PUBMED, y la RED COCHRANE, considerando el Registro Cochrane de ensayos clínicos, para identificar ensayos controlados prospectivos de la evaluación de baño preoperatorio con clorhexidina versus placebo o ningún baño para prevenir la infección del sitio quirúrgico.

Se valoró las medidas de resultados de las relaciones de riesgo RR con la medida de ratio OR, calculados utilizando un modelo de efectos aleatorios DerSimonian-Laird y un modelo de efectos dicotómica MantelHaenszel.

Se obtuvo 16 ensayos que cumplieron los criterios de inclusión; el total de pacientes fue de 17932 pacientes, de los cuales 7952 recibieron un baño con clorhexidina y 9980 pacientes fueron asignados a diferentes grupos de comparación.

En general, en el grupo de clorhexidina, $6,8 \%$ de los pacientes desarrolló infección del sitio quirúrgico en comparación con el 7,2\% de los pacientes en el grupo de comparación, quienes usaron placebo o nada.

Dentro de las principales conclusiones se colige que el baño con clorhexidina no redujo significativamente la incidencia global de infección del sitio quirúrgico en comparación con jabón, placebo o sin ducha (riesgo relativo:0,90, intervalo de confianza del 95\%: 0,77 a 1,05, p =0,19); sin embargo, los autores cuestionan que muchos de los estudios incluidos en el metaanálisis omiten el protocolo de aplicación de la clorhexidina, la concentración, la frecuencia, exclusión que puede generar sesgos en los resultados.

\section{DISCUSIÓN}

La piel es la fuente principal de gérmenes patógenos causantes de las infecciones del campo quirúrgico (Maimone, 2009); por tanto, disminuir la carga de gérmenes patógenos representan por sí una disminución en el riesgo de presentar infecciones de sitio quirúrgico y, a su vez, una reducción de las principales causas de morbimortalidad entre los pacientes quirúrgicos, de la estancia hospitalaria y de los costes de atención, lo cual implica una atención segura para el paciente. 
Dado lo anterior, es necesario preparar la piel de los pacientes antes de ser sometida a cirugía, como lo recomienda las guías del Center for Disease Prevention and Control las cuales indican, entre otras recomendaciones, el baño prequirúrgico y la preparación preoperatoria de la piel con un antiséptico de probada eficacia (CDC, 1999).

La clorhexidina es una biguanida catiónica desarrollada en Inglaterra en 1954, usada como antiséptico. La actividad antimicrobiana es atribuida a su unión y a la disrupción de la membrana citoplásmica, procesos que alteran el equilibrio osmótico y precipitan los contenidos celulares (Maya, 2011).

En cuanto a su uso, Maimone considera que "el baño prequirúrgico con gluconato de clorhexidina ha reducido la colonización de la piel" (2009, p.15). Según Ayestarán (2012), la asociación de clorhexidina y alcohol mejoran la eficacia dado que se complementa la rapidez de acción del alcohol con la acción residual de la clorhexidina $(\mathrm{CH})$, además de que parece potenciar la capacidad de la $\mathrm{CH}$ para penetrar hasta el estrato córneo de la piel y lograr el efecto remanente.

Como antiséptico prequirúrgico de primera elección, la clorhexidina se considera la mejor opción, así lo confirman Dizer et al (2009), quienes sostienen que un baño de clorhexidina excluyendo el área de la cabeza, antes de una operación, dos veces en el periodo preoperatorio, es útil para reducir la infección del sitio quirúrgico en dicho periodo.

En la presente investigación, aunque los artículos no son con población infantil, brindan datos relevantes acerca de su uso y sus efectos a largo plazo, en la preparación prequirúrgica de la piel inclusive, Chlebicki, Safdar, O'Horo y Maki (2013), en el metaanálisis de ensayos clínicos disponibles, sostienen que su uso no sugiere ninguna ventaja apreciable en cuanto a prevenir infección del sitio quirúrgico. Sin embargo, la mayoría de los estudios omiten detalles sobre la aplicación de la clorhexidina, debido a que, según los mismos autores, la mayoría de los estudios no proporcionan datos específicos acerca de la proporción de pacientes que en cada grupo recibió antibióticos perioperatorios, lo cual oculta el verdadero efecto beneficioso del uso de la clorhexidina preoperatorio, además de que el uso de la sustancia puede haber sido menos óptimo en varios estudios. En todos los ensayos, los pacientes fueron instruidos para simplemente bañarse o ducharse con el agente de la manera habitual: en específico, no se indicó cómo mantener la clorhexidina en la piel durante varios minutos antes de lavarla para alcanzar el efecto; por consiguiente, se sugiere un mejor diseño para los ensayos en cuanto a que incluyan una duración determinada y frecuencia de exposición a la clorhexidina para determinar si el baño preoperatorio con clorhexidina realmente reduce infección del sitio quirúrgico.

Por el contrario, Dizer, Hatipoglu, Kaymakcioglu, Tufan, Yava, Iyigun \& Senses (2009), en sus resultados mencionan que en el grupo en el que no se aplicó baño de clorhexidina, el riesgo de infección fue mayor 4.76 veces $(95 \% \mathrm{CI}=1.20-18.83)$, incluso, después de las correcciones por edad y género. La diferencia entre ambas investigaciones es que la preparación de la piel la última, fue ejecutada por un profesional en enfermería.

Es necesario investigar más sobre las concentraciones específicas y sus efectos en niños (as), dado que no es clara cuál es la concentración adecuada; al respecto Stinner, Krueger, Masini y Wenke (2011) comentan que en estudios in vitro la dilución de la clorhexidina se correlaciona directamente con la actividad bactericida y su eficacia se relaciona de manera directa con su tiempo de contacto: basándose en sus resultados, los autores 
recomiendan el uso de clorhexidina al 4\% para la preparación del sitio quirúrgico y permitir un mínimo de 2 minutos de tiempo de contacto antes de realizar la incisión en la piel. A pesar de que el presente estudio precisaba de investigaciones con población estudiantil, es necesario agregar que los productos con clorhexidina no están aprobados por la (FDA) en caso de niños (as) menores de 2 meses y están contraindicados en pacientes neonatos con bajo peso al nacer (Maimone, 2009).

En cuanto a la frecuencia, si bien todos los estudios varían en cuanto al número de veces que se debe aplicar la clorhexidina, todos coinciden en que deben ser más de dos veces antes de la cirugía (Chlebicki, Safdar, O’Horo y Maki. 2013, p.170).

En lo que respecta a los efectos secundarios, en solo un ensayo de 15 se reportó efectos adversos del lavado preoperatorio o el baño con clorhexidina. En este ensayo, en cinco pacientes se produjo picazón o enrojecimiento de la piel $(0,36 \%)$, malestares que también se presentaron en cinco integrantes del grupo placebo $(0,36 \%)$, mas no se observó efectos adversos atribuidos a la ducha o el baño con clorhexidina, mencionados en los otros 15 ensayos restantes (Chlebicki, Safdar, O’Horo y Maki, 2013); por ende, no se considera un efecto común.

Debido a las carencias de información específica, urge desarrollar una investigación primaria específica, en la población pediátrica, para evaluar la seguridad en los niños (as) y especificar el protocolo en cuanto al uso de la clorhexidina, en concentraciones, frecuencia, duración en contacto con la piel, y la educación a los padres si es necesario que fuese domiciliaria. Además se requiere mayor claridad en la metodología empleada para evitar sesgos en los datos presentados.

Por otra parte, es urgente generar estrategias para disminuir la incidencia de infecciones en el sitio quirúrgico, lo cual fundamenta la necesidad de que sea una enfermera profesional la que prepare al paciente para la intervención, debido a que cuenta con el conocimiento sobre la rigurosidad del procedimiento para disminuir los efectos adversos.

Por otro lado, como lo mencionan los autores consultados, se requiere investigación primaria de mejor calidad que aporte evidencia de peso acerca de los beneficios de esta práctica.

\section{CONCLUSIONES}

No se encontró suficiente evidencia para responder de forma contundente a la pregunta clínica planteada sobre el uso de la clorexidina al $2 \%$ en la población pediátrica para la preparación preoperatoria de la zona quirúrgica, para prevenir la infección. Existe evidencia científica de que el baño con clorhexidina al $4 \%$ es efectivo en adultos; no obstante, en niños (as) no es absolutamente clara la concentración apropiada para alcanzar el mismo efecto. Por lo cual se requieren de investigación primaria específicamente en niños (as). 


\section{Revista Electrónica Enfermería Actual en costa Rica}

\section{BIBLIOGRAFÍA}

Aizcorbe, J., Izco, T., De la Torre, A., Usoz, J., Soto J. (2000). Vigilancia y control de la infección de sitio quirúrgico. [Versión Electrónica]. ANALES Sis San Navarra, 23 (Supl. 2), 129-141. Recuperado de http://www.cfnavarra.es/salud/anales/textos/vol23/suple2/suple12.html

Alemán, L. y Guanche, H. (ene-mar, 2001). Etiología de la infección del sitio quirúrgico en pacientes egresados del Hospital Clinicoquirúrgico Docente "Joaquín Albarrán". Rev. Cubana, 40 (4), 291-296. Recuperado de http://scielo.sld.cu/scielo.php?script=sci_arttext\&pid=S0034-74932001000400009\&lng=es.

Ayestarán, A. (2012). Clorhexidina al 2\% en la desinfección del campo quirúrgico. Informe para la Comisión de Infecciones y Farmacia y Terapéutica del Hospital de Barcelona. Recuperado de http://gruposdetrabajo.sefh.es/genesis/genesis/Documents/Clorhexidina alcohol desinf campo quirurgico HBA 03_2012.pdf

Burke, H., Derek, B., BAO, J., Ni-Dhonochu, T., Shields, W., Desmond, C. y Mealy, K. (august, 2010). Preoperative Hypoalbuminemia is an Independent Risk Factor for the Development of Surgical Site Infection Following Gastrointestinal Surgery: A Multi-Institutional Study [Versión Electrónica]. Annals of Surgery, 252 (2), 325-329. doi: 10.1097/SLA.0b013e3181e9819

Caja Costarricense de Seguro Social (2006). Comité de Control y Prevención de Infecciones Nosocomiales. San José: Hospital Dr. Rafael Ángel Calderón Guardia.

Canton, E., Viudes, A. y Pemán, J. (2001). Infección sistémica nosocomial por levaduras. Rev. Iberoam. Micol, $18,51-55$.

(s.a.). (2013). Centers for Disease Control and Prevention. Recuperado de http://www.cdc.gov/

Chlebicki, M., Safdar, N., O'Horo, J., Maki, D. (2013). Preoperative chlorhexidine shower or bath for prevention of surgical site infection: a meta-analysis. American Journal of Infection Control, 41(2),167-73. doi: 10.1016/j.ajic.2012.02.014

Dizer, B., Hatipoglu, S., Kaymakcioglu, N., Tufan, T., Yava, A., Iyigun, E. y Senses, Z. (2009). The effect of nurse-performed preoperative skin preparation on postoperative surgical site infections in abdominal surgery. Journal Clinical Nurse, 18(23),3325-32. doi: 10.1111/j.1365-2702.2009.02885.x.

Harris, R., Helfand, M., Woolf, S., Lohr, K., Mulrow, C., Teutsch, S. y Atkins, D., (2001). Preventive Services Task Force. Current methods of the U.S. Preventive Services Task Force: a review of the process. Am. J. Prev. Med.,20(3S), 21-35.

Maimone, S. (octubre, 2009). Gluconato de Clorhexidina: ¿el mejor antiséptico para la piel? ECI, 1(2), 60-63 Recuperado de http://www.adeci.org.ar/LinkClick.aspx?fileticket=IWcO819BCtQ\%3D\&tabid=75 
Mangram, A., Horan, T., Pearson, M., Silver, L., Jarvis, W. (1999). Guideline for prevention of surgical site infection. Centers for Disease Control and Prevention. CDC.

Maya, J., Ruiz, S., Pacheco, R., Valderrama, L., Villegas, M. (junio, 2011). Papel de la clorhexidina en la prevención de las infecciones asociadas a la atención en salud. Infect., 15(2), 98-107. Recuperado de http://www.scielo.org.co/scielo.php?script=sci_arttext\&pid=S0123-93922011000200004\&lng=es.

Murray, M., Saltzman, Gryzlo, S., Terry, M., Woodward, C., Nuber, G. (mayo, 2011).Efficacy of preoperative home use of $2 \%$ chlorhexidine gluconate cloth before shoulder surgery. Journal Shoulder Elbow Surgery, 20(6), 928-33. doi: 10.1016/j.jse.2011.02.018.

Pujol, M. y Limón, E. (enero, 2013). Epidemiología general de las infecciones nosocomiales. Sistemas y programas de vigilancia. Revista Enfermedades Infecciosas y Microbiología Clínica., 31(2), 108-113.

Red Critical Appraisal Skills Programme Español. CASPe. Recuperado de http://www.redcaspe.org/drupal/. Copyright (C) 2013

Rojas L., Cubero, C. y Rodríguez, A. (2013) Enfermería basada en la Evidencia. San José: CIEBE-CR.

Silva, P. (2013). An evidence based protocol for preoperative preparation. J Perioper Pract., 23(4), 87-90. PMID: 23691885 [PubMed - in process]

Stinner, D., Krueger, C., Masini, B., Wenke, J. (octubre, 2011). Time-dependent effect of chlorhexidine surgical prep. J Hosp Infect, 79(4), 313-6. doi: 10.1016/j.jhin.2011.08.016. 